\title{
Elevating Twitter-Based Journal Club Discussions by Leveraging a Voice-Based Platform: \#HemepathJC Meets Clubhouse
}

\author{
Aadil Ahmed $^{1}$ (D) Kamran M. Mirza ${ }^{2} \cdot$ Sanam Loghavi ${ }^{3}$
}

Accepted: 9 July 2021 / Published online: 29 September 2021

(c) The Author(s), under exclusive licence to Springer Science+Business Media, LLC, part of Springer Nature 2021

\begin{abstract}
Purpose of Review Social media-based scientific journal clubs provide an opportunity to promote published literature to a broader audience and allow robust multi-disciplinary and inter-professional discussion. Hematopathology Journal Club (\#HemepathJC) on Twitter has successfully conducted monthly sessions since November 2019, covering topics related to lymphoma and leukemia.

Recent Findings To enhance connectivity, multitasking, and productivity, we present our experience of leveraging the voicebased platform Clubhouse concurrent with Twitter.

Summary The Twitter and Clubhouse partnership for \#hemepathJC holds the potential to increase dissemination of scientific knowledge and further promote journal club format discussion.
\end{abstract}

Keywords HemepathJC $\cdot$ Hematopathology $\cdot$ Online journal club $\cdot$ Social media $\cdot$ Twitter $\cdot$ Clubhouse

Social media-based platforms such as Twitter offer an easyto-use, free, and easily accessible global communication tool that can be leveraged for uninterrupted information exchange in the medical world. Devoid of traditional barriers to knowledge-sharing, Twitter allows for free, live, 24/7/365 real-time learning [1, 2]. In malignant hematology/hematopathology discourse, tweetorials, conference-related tweets, \#SoMe based multi-institutional collaborations, and online journal clubs have, until recently, been considered the new kids on the block [3, 4]. The latest member of this gang is Clubhouse, a voice-based social network where people from around the globe can come together to chat, listen, or both with others in real time. The layout of Clubhouse is very

This article is part of the Topical Collection on Social Media Impact of Hematologic Malignancies

Aadil Ahmed

aadil_ahmed@rush.edu

1 Department of Pathology \& Laboratory Medicine, Rush University Medical Center, Chicago, IL, USA

2 Department of Pathology \& Laboratory Medicine, Loyola University Health System, Maywood, IL, USA

3 Department of Hematopathology, MD Anderson Cancer Center, Houston, TX, USA user friendly (Fig. 1) allowing for easy visual identification of who is speaking and who is in the room, with easy access for all to be allowed to speak with a click of an icon. In this sense, Clubhouse is similar to TwitterSpaces and the main similarities and differences are summarized in Table 1.

The online-only hematopathology journal club, @ HemepathJC (\#HemepathJC), was initiated on Twitter, a microblogging social networking service on which users post and interact with messages known as "tweets" in November 2019. This venture extends the idea of academic journal clubs to a global audience and has continued with monthly journal club sessions since. A few months later, in April 2020, Clubhouse was launched. Clubhouse is a novel voicebased social network, originally launched in the Fall of 2019 and released for iOS operating systems in March 2020, with over 10 million active users as of February 2020, where a global audience can come to talk, listen, and learn from each other in real time. We decided to leverage this voice-based platform to facilitate the HemepathJC process, and in March 2021, the Twitter-and-Clubhouse combined HemepathJC was born. This unique joint experience combined microblogging (Twitter) with voice-based discussion (Clubhouse) for the first time. We report the implementation of this novel online journal club experience which had a truly international and multidisciplinary audience using multiple social 


\section{HemepathJC}

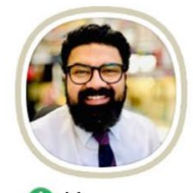

* Kamran

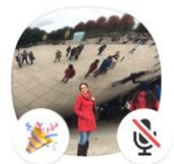

Mehrnoosh

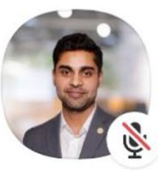

Aadil

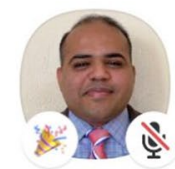

Ashwin

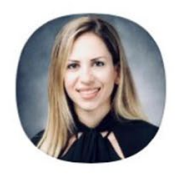

Sanam

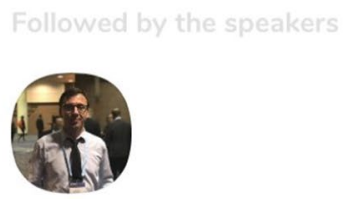

Ahmad

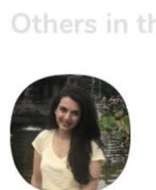

Samaneh
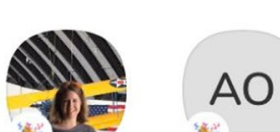

Leave quietly

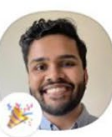

MJ

(F)

Majd

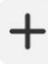

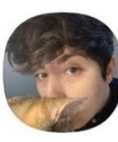

Lo

Fig. 1 Screenshot from Clubhouse app during \#hemepathJC showing simplicity of layout. Speakers are seen in top section, users followed by speakers in middle section, and those perusing the room (but not speaking) seen at the bottom. The green icon next to Kamran (top right) indicates he was speaking at the time of the screengrab. The mute icon next to Aadil (top row, middle user) indicates that his mic is muted

media platforms concurrently. The experience was highly successful, and we believe that this hybrid format will be a model for other journal clubs to follow.

The evolution of the academic "journal club" (JC) has a long and rich history which shows increasing modernity, communication, and adaptability over time [5]. With a continually expanding global outreach online, Twitter has become one of the most common platforms of choice for JCs with over 27 active twitter JCs within the medical specialties of which 4 relate to the field of pathology [6]. HemepathJC, a hematopathology-directed JC, has conducted 14 monthly sessions to date, alternating between topics related to leukemia (\#leusm) and lymphoma (\#lymsm). The journal club account (@HemepathJC) has garnered > 1400 followers from around the globe and attracts hematopathologists, hematology-oncology clinicians, and basic science researchers during JC hour. This Twitter-based JC has provided the opportunity to discuss and share perspectives, outcomes, and updates based on different practice settings while offering a platform to network and collaborate.

Twitter has proven to be an efficient medium for pathology education and in particular hematopathology [7, 8, 9•]. Given a significant hematopathology audience on Twitter, HemepathJC gets considerable attendance during the monthly JC hour; however, limitations like evening hours on a weekday, different time zones, and lack of technical aptitude (quick typing, microblogging) may theoretically hinder participation of some participants. Added to this, COVID-19 restrictions brought forward an additional need for connectivity at an emotional level (verbal, voice). We sought to address whether the launch of the Clubhouse application on iOS (Apple Inc., Cupertino, CA) could be introduced as an additional tool for discussion in our JC activity and chose to launch the first of this type of Twitter-Clubhouse JC in March 2021. This inaugural JC registered 16 participants on Clubhouse with about half of them being brand new to the JC. The participants included medical students, trainees, and hematopathology faculty from both academia and community practice. The format consisted of a live discussion between the moderator and the first author of the chosen paper, followed by questions from the participants. The author discussed the immunophenotypic manifestations

Table 1 Comparison of Twitter, Twitter Spaces, and Clubhouse

\begin{tabular}{lll}
\hline Twitter & Twitter Spaces & Clubhouse \\
\hline Independent app & Feature of Twitter, similar to fleets & Independent app \\
Available on mobile and desktop devices & Only available on mobile & Only available on mobile \\
Available to both Android and iOS users & Twitter spaces beta available to both Android and iOS users & iOS-only app at this time \\
Not invitation-based & Not invitation-based & Currently invitation-only \\
Need to follow users to see content & Relies on Fleets posted by those a user follows & Allows perusing feeds \\
& & of all available public \\
& & rooms \\
\hline
\end{tabular}


of persistence clonal hematopoiesis in NPMI-mutated acute myeloid leukemia in the setting of post-remission and guided the audience about separating it from measurable residual disease (MRD) [10]. The Twitter live feed was concurrently populated by HemepathJC organizers and participants for the audience limited to Twitter. Any questions on Twitter live feed were also conveyed to the first author and were answered live and subsequently responded on Twitter. A total of 211 tweets by 44 participants, generating over 1 million impressions, were recorded on Twitter during and in the few days after this combined hemepathJC.

While this objective data is encouraging, our subjective impression was no different. The purpose of JC is to have an engaged audience and stimulate discussion which was successfully fulfilled on the voice-based platform in an efficient manner. While Twitter has provided flexibility and permanence of tweets, it also requires continued engagement to update the tweets and active typing to respond during the dedicated hour. The voice-based platform on the other hand provides an opportunity to listen and submit an audio response while one may perform other tasks as well. The productivity and amount of information disseminated via audio response are, in many ways, superior and faster. Not to mention that voice-based communication could possibly create a stronger bond and emotional connection [11]. The personability of our participants was felt by warm introductions and lively chat in an informal setting, thereby bringing the human touch as an antidote to COVID isolation.

The successful implementation of this mode of JC is a model other JCs can follow. Currently, a major limitation is that Clubhouse is an invitation-only, iOS-exclusive chat app with exclusivity that is less desirable for the purpose of a broad educational activity such as JC. However, the reach of Clubhouse should be much broader soon when it expands beyond an invitation-only iOS-based platform. Once it opens to a broader audience, a hybrid model using both text and voice-based applications, including microblogging (and even Twitter Spaces) in the future seems to promise robust JC discussions, connectivity, and excellence, not only for online journal club discourse but as a supplement to in-person or hybrid learning at major national meetings such as ASCO, ASH, USCAP, and EHA, similar to how Twitter has supplemented learning at these conferences [12, 13]. In the post-pandemic world, with a re-evaluation of the frequency of in-person conversations, could Clubhouse provide a voice-based collaboration tool which we incorporate with the knowledge of the pros, cons, challenges, and pitfalls we already have the benefit of learning from, from Twitter? Could Clubhouse-based \#FOAMed parallel the trajectory of Twitter? Clubhouse-based multi-institutional research and collaboration allow for increased connectivity between global physicians, scientists, and researchers in the way Twitter did? We definitely think so.

\section{Declarations}

Human and Animal Rights and Informed Consent This article does not contain any studies with human or animal subjects performed by any of the authors.

Conflict of Interest None.

\section{References}

Papers of particular interest, published recently, have been highlighted as:

- Of importance

1. Banerjee R, Kelkar AH, Logan AC, Majhail NS, Pemmaraju $\mathrm{N}$. The democratization of scientific conferences: Twitter in the era of COVID-19 and beyond. Curr Hematol Malig Rep. 2021;16(2):132-9.

2. Mukhopadhyay S, Kanakis C, Golab K, Hermelin D, Crane GM, Mirza KM. The network that never sleeps. Lab Med. 2021;lmaa113. https://doi.org/10.1093/labmed/lmaa113.

3. Pemmaraju N, Thompson MA, Qazilbash M. Disease-specific hashtags and the creation of Twitter medical communities in hematology and oncology. Semin Hematol. 2017;54(4):189-92.

4. Attai DJ, Anderson PF, Fisch MJ, Graham DL, Katz MS, Kesselheim J, Markham MJ, Pennell NA, Sedrak MS, Thompson MA, Utengen A, Dizon DS. Risks and benefits of Twitter use by hematologists/oncologists in the era of digital medicine. Collaboration for Outcomes on Social Media in Oncology (COSMO). Semin Hematol. 2017;54(4):198-204.

5. Topf JM, Sparks MA, Phelan PJ, Shah N, Lerma EV, GrahamBrown MPM, Madariaga H, Iannuzzella F, Rheault MN, Oates $\mathrm{T}$, Jhaveri KD, Hiremath S. The evolution of the journal club: from Osler to Twitter. Am J Kidney Dis. 2017;69(6):827-36.

6. Stoneman S, Hiremath S. Twitter-based journal clubs: bringing critical appraisal to the social table. Semin Nephrol. 2020;40(3):264-72.

7. Mukhopadhyay S, Booth AL, Calkins SM, Doxtader EE, Fine SW, Gardner JM, Gonzalez RS, Mirza KM, Jiang XS. Leveraging technology for remote learning in the era of COVID-19 and social distancing. Arch Pathol Lab Med. 2020;144(9):1027-36.

8. El Hussein S, Lyapichev KA, Crane GM, Mirza KM, Pemmaraju N, Medeiros LJ, Khoury JD, Loghavi S. Social media for hematopathologists: medical practice reinvented-\#Hemepath. Curr Hematol Malig Rep. 2020;15(5):383-90.

9. El Hussein S, Khoury JD, Lyapichev KA, Tashakori M, Khanlari M, Miranda RN, Kanagal-Shamanna R, Wang SA, Ahmed A, Mirza KM, Crane GM, Medeiros LJ, Loghavi S. Next-generation scholarship: rebranding hematopathology using Twitter: The MD Anderson Experience. Mod Pathol. 2020;1-8. The editorial describes the importance of social media and especially Twitter in disseminating hematopathology knowledge.

10. Loghavi S, DiNardo CD, Furudate K, Takahashi K, Tanaka T, Short NJ, Kadia T, Konopleva M, Kanagal-Shamanna R, Farnoud NR, Pierce S, Khoury JD, Jorgensen JL, Patel KP, Daver N, Yilmaz M, Medeiros LJ, Kantarjian H, Ravandi F, Wang SA. Flow cytometric immunophenotypic alterations of persistent clonal haematopoiesis in remission bone marrows of patients with NPM1-mutated acute myeloid leukaemia. Br J Haematol. 2021;192(6):1054-63. 
11. Kumar A, Epley N. It's surprisingly nice to hear you: Misunderstanding the impact of communication media can lead to suboptimal choices of how to connect with others. J Exp Psychol Gen. 2021;150(3):595-607.

12. Pemmaraju N, Thompson MA, Mesa RA, Desai T. Analysis of the Use and Impact of Twitter During American Society of Clinical Oncology Annual Meetings From 2011 to 2016: Focus on Advanced Metrics and User Trends. J Oncol Pract. 2017 Jul;13(7):e623-e631. https://doi.org/10.1200/JOP.2017.021634.
13. Pemmaraju N, Mesa RA, Majhail NS, Thompson MA. The use and impact of Twitter at medical conferences: best practices and Twitter etiquette. Semin Hematol. 2017;54(4):184-8.

Publisher's Note Springer Nature remains neutral with regard to jurisdictional claims in published maps and institutional affiliations. 